\title{
Nationwide population genetic screening improves outcomes of newborn screening for hearing loss in China
}

\author{
Qiuju Wang, $M D^{1}$, Jiale Xiang, $\mathrm{MS}^{2}$, Jun Sun, $\mathrm{PhD}^{3,4}$, Yun Yang, $\mathrm{PhD}^{2}$, Jing Guan, $\mathrm{MD}^{1}$, \\ Dayong Wang, $\mathrm{MD}^{1}$, Cui Song, $\mathrm{MD}^{5,6}$, Ling Guo, $\mathrm{BS}^{7}$, Hongyang Wang, $\mathrm{MD}^{1}$, Yaqiu Chen, $\mathrm{BS}^{8}$, \\ Junhong Leng, $\mathrm{PhD}^{8}$, Xiaman Wang, $\mathrm{BS}^{9}$, Junqing Zhang, $\mathrm{BS}^{3}$, Bing Han, $\mathrm{MD}^{1}$, Jing Zou, $\mathrm{MS}^{10^{\prime}}$, \\ Chengbin Yan, $\mathrm{MS}^{2}$, Lidong Zhao, MD', Hongyu Luo, $\mathrm{MS}^{2}$, Yuan Han, $\mathrm{BS}^{11}$, Wen Yuan, $\mathrm{MS}^{11}$, \\ Hongyun Zhang, $\mathrm{MS}^{9}$, Wei Wang, $\mathrm{PhD}^{12}$, Jian Wang, $\mathrm{PhD}^{13,14}$, Huanming Yang, $\mathrm{PhD}^{13,14}$ \\ Xun Xu, PhD ${ }^{13,15}$, Ye Yin, MS ${ }^{2}$, Cynthia C. Morton, PhD ${ }^{5,16,17}$, Lijian Źhao, BS ${ }^{9}$, Shida Zhu, MS $\oplus^{13,15,18}$, \\ Jun Shen, $\mathrm{PhD}^{5}$ and Zhiyu Peng, $\mathrm{PhD}{ }^{2}$
}

\begin{abstract}
Purpose: The benefits of concurrent newborn hearing and genetic screening have not been statistically proven due to limited sample sizes and outcome data. To fill this gap, we analyzed outcomes of newborns with genetic screening results.
\end{abstract}

Methods: Newborns in China were screened for 20 hearingloss-related genetic variants from 2012 to 2017. Genetic results were categorized as positive, at-risk, inconclusive, or negative. Hearing screening results, risk factors, and up-to-date hearing status were followed up via phone interviews.

Results: Following up 12,778 of 1.2 million genetically screened newborns revealed a higher rate of hearing loss by three months of age among referrals from the initial hearing screening $(60 \%$ vs. $5.0 \%, P<0.001)$ and a lower rate of lost-to-follow-up/documentation ( $5 \%$ vs. $22 \%, P<0.001)$ in the positive group than in the inconclusive group. Importantly, genetic screening detected $13 \%$ more hearing-impaired infants than hearing screening alone and identified $2,638(0.23 \%$ of total $)$ newborns predisposed to preventable ototoxicity undetectable by hearing screening.

Conclusion: Incorporating genetic screening improves the effectiveness of newborn hearing screening programs by elucidating etiologies, discerning high-risk subgroups for vigilant management, identifying additional children who may benefit from early intervention, and informing at-risk newborns and their maternal relatives of increased susceptibility to ototoxicity.

Genetics in Medicine (2019) 21:2231-2238; https://doi.org/10.1038/s41436019-0481-6

Keywords: genetic screening; hearing screening; hearing loss; newborns; clinical benefits

\section{INTRODUCTION}

Hearing loss is one of the most common birth defects with lifelong impact that may be ameliorated by early detection and intervention. ${ }^{1}$ Newborn hearing screening programs (NBHS) have been globally adopted since the 1990s. They serve as a primary approach to detecting hearing loss in neonates and triggering early intervention. ${ }^{2}$ As designed, conventional newborn hearing screening neither detects mild hearing loss nor offers prognosis to delayed-onset or drug- induced hearing loss. ${ }^{2-5}$ Hearing screening is unable to elucidate the etiology of hereditary hearing loss, which accounts for at least $50 \%$ to $60 \%$ of childhood hearing loss. ${ }^{5}$ Furthermore, high rates of lost-to-follow-up/documentation (LTF/D) lessen the effectiveness of NBHS. ${ }^{6}$

Limited genetic screening of a small number of genes commonly associated with hearing loss (GJB2, SLC26A4, and MT-RNR1) to improve the detection of late-onset prelingual hearing loss was first proposed in 2006 (ref. ${ }^{5}$ ).

${ }^{1}$ Department of Otolaryngology-Head and Neck Surgery, Chinese PLA Institute of Otolaryngology, Chinese PLA General Hospital, Beijing, China; ${ }^{2}$ BGI Genomics, BGI-Shenzhen, Shenzhen, China; ${ }^{3}$ Tianjin Medical Laboratory, BGI-Tianjin, BGI-Shenzhen, Tianjin, China; ${ }^{4}$ Binhai Genomics Institute, BGI-Tianjin, BGI-Shenzhen, Tianjin, China; ${ }^{5}$ Brigham and Women's Hospital, Harvard Medical School, Boston, MA, USA; ${ }^{6}$ Children's Hospital of Chongqing Medical University, Chongqing, China; ${ }^{7}$ Jining Maternal and Child Health Care Service Center, Jining, China; ${ }^{8}$ Tianjin Women and Children's Health Centre, Tianjing, China; ${ }^{9}$ BGI Clinical Laboratory, BGI-Shenzhen, Shenzhen, China; ${ }^{10}$ MGI, BGI-Shenzhen, Shenzhen, China; ${ }^{11}$ Wuhan BGI Clinical Laboratory, BGI-Shenzhen, Wuhan, China; ${ }^{12}$ BGI-Beijing, BGI-Shenzhen, Beijing, China; ${ }^{13}$ BGI-Shenzhen, Shenzhen, China; ${ }^{14}$ James D. Watson Institute of Genome Sciences, Hangzhou, China; ${ }^{15}$ China National GeneBank, BGI-Shenzhen, Shenzhen, China; ${ }^{16}$ Manchester Center for Audiology and Deafness, School of Health Sciences, University of Manchester, Manchester, UK; ${ }^{17}$ Broad Institute of Harvard and MIT, Cambridge, MA, USA; ${ }^{18}$ Shenzhen Engineering Laboratory for Innovative Molecular Diagnostics, Shenzhen, China. Correspondence: Lijian Zhao (zhaolijian@bgi.com) or Shida Zhu (zhushida@genomics.cn) or Jun Shen (jshen5@bwh.harvard.edu) or Zhiyu Peng (pengzhiyu@bgi.com)

These authors contributed equally: Qiuju Wang, Jiale Xiang, Jun Sun and Yun Yang, Jing Guan, Dayong Wang, and Cui Song 
Targeted screening of newborns for pathogenic variants in these genes represents an affordable model. Studies have demonstrated the feasibility of concurrent hearing and genetic screening. ${ }^{7-11}$ However, the benefits of such practices have not been quantified due to limited sample sizes and outcome data. Herein we report results of genetic screening on $\sim 1.2$ million newborns in China and outcomes of 12,778 infants with genetic findings followed up via phone interviews.

\section{MATERIALS AND METHODS}

\section{Study design}

This study was performed with the approval of the Institutional Review Board of BGI.
Newborns were enrolled nationwide in China who received genetic screening from March 2012 to September 2017. Families or guardians of subjects with at least one variant identified between March 2012 and December 2016 were targeted for follow-up phone interviews from November 2016 to March 2017 (first interview period). Given the relatively small number of subjects with biallelic variants, additional subjects with biallelic autosomal or homoplasmic mitochondrial variants identified from January to September 2017 were interviewed between December 2017 and January 2018 (second interview period) to increase the power of the study (Fig. 1). Subjects with biallelic variants who reported no hearing loss during the first interview period were recontacted during the second interview period. Newborns without any

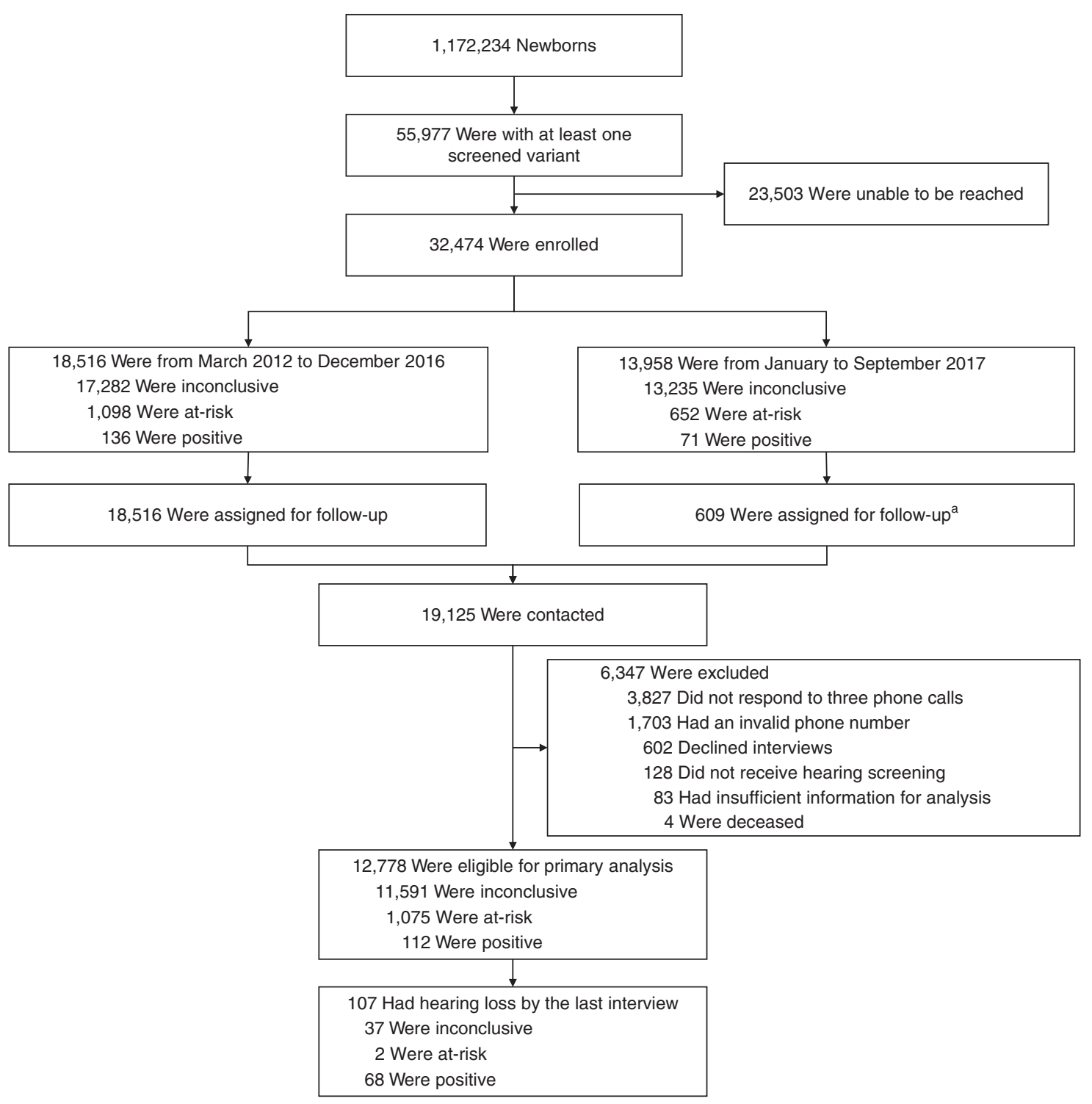

Fig. 1 Enrollment and outcomes of subjects participating in follow-up interviews. Genetic screening results were classified as follows: positive (biallelic variants in either GJB2 or SLC26A4), inconclusive (a heterozygous variant in GJB2 or SLC26A4, or presence of any GJB3 variant), at-risk (any mitochondrial variant), and negative (no variant). ${ }^{a}$ Considering limited samples size of subjects with biallelic variants from 2012 to 2016 , we initiated interviews for a new group of subjects with biallelic autosomal or homoplasmic mitochondrial variants from January to September 2017, with subjects with positive ( $n=71$ ), homoplasmic at-risk $(n=536)$, and homozygous GJB3 $(n=2)$ results targeted for follow-up. 
variant were not followed up because clinical management remains the same as the standard of care (Supplementary Figure S1 online).

\section{Concurrent newborn genetic and hearing screening}

Newborn hearing screening and sampling of blood spot specimens for genetic screening were conducted concurrently as inpatients after birth. Parental informed consent was obtained for each newborn to have the newborn hearing and genetic screening.

\section{Newborn genetic screening}

Newborn genetic screening was offered to families of newborns in participating hospitals nationwide. The cost per genetic screening test differed by regions, ranging from US $\$ 32$ to US $\$ 48$, which covered the administrative cost of organizing the screening and data systems, labor and material cost for consenting, specimen collection, shipping and handling, quality assurance, laboratory assays, and result disclosure. Blood spot specimens were obtained via heel stick onto filter paper according to the routine newborn screening protocol and dried at room temperature prior to being delivered to BGI's clinical laboratories for testing. Turnaround time for processing was around five business days following receipt of the dried blood spot specimen. Results were generally available by two to four weeks of age. Families were notified via text messages of the availability of results and instructions on obtaining clinical reports at designated hospitals. Since 2017 families could use the online BGI Inquiry and Notification System to track the status of the test. Upon obtaining clinical reports of the genetic screening results from the hospital, genetic counseling was offered to those with any genetic variant. For newborns with a high risk of hereditary hearing loss, basic genetic knowledge of inheritance modes, hereditary hearing loss, and avoiding aminoglycoside exposure (for MT-RNR1 variant carriers) were provided in person.

The limited genetic screening entailed genotyping 20 variants in GJB2, SLC26A4, MT-RNR1, and GJB3 (Supplementary Table S1 online) by matrix-assisted laser desorption/ ionization time of flight (MALDI-TOF) mass spectrometry as previously validated and reported. ${ }^{7,9}$ Genetic screening results were classified as follows: positive (biallelic variants in either GJB2 or SLC26A4), inconclusive (a heterozygous variant in GJB2 or SLC26A4, or presence of any GJB3 variant), at-risk (any mitochondrial variant), and negative (none of the 20 screened variants identified). All homozygous results were confirmed by Sanger sequencing. Heterozygous results were confirmed by repeating the MALDI-TOF assay. Sanger sequencing was performed if results of the original and repeat runs were inconsistent.

Each subject was categorized into only one group based on the genetic results. When a subject's results fell into more than one category, the subject was assigned to a group in the following order of priority: positive, at-risk, inconclusive, and negative.

\section{Newborn hearing screening}

Newborn hearing screening results were collected via phone interviews. The initial hearing screening was typically conducted using otoacoustic emission (OAE) by a trained nurse in the birth hospital 48-72 hours after birth in China. Newborns who did not pass the initial hearing screening were referred for retests around 42 days of age when $\mathrm{OAE}$ and/or automated auditory brainstem response (AABR) tests were performed. Newborns who did not pass the 42-day retest were referred to comprehensive diagnostic audiometry conducted by audiologists generally around three months of age (Supplementary Figure S1 online).

\section{Phone interview}

Newborns with a non-negative genetic result were followed up via phone interviews, during which self-reported hearing screening results, hearing loss risk factors, and up-to-date hearing status were collected. Subjects were considered LTF/D if they were referred from the initial inpatient hearing screening but did not follow through with either the 42-day retest or the three-month audiologic evaluation. A subject was considered to have hearing loss if (1) a formal audiologic evaluation determined a hearing threshold above 25 decibels, (2) hearing rehabilitation had been implemented (e.g., hearing aids and cochlear implants), or (3) hearing loss was reported based on behavioral assessment. The severity of hearing loss was graded according to the World Health Organization's standards for children, namely profound $(\geq 81$ decibels), severe (61-80 decibels), moderate (31-60 decibels), and mild/ slight (26-30 decibels). ${ }^{12}$ The degree of hearing loss was recorded as "unspecified" if audiologic evaluation results were not available.

\section{Statistical analysis}

Subjects with sufficient information obtained from phone interviews were included for the primary analysis. For categorical data, summary data were reported as frequencies and percentages, and chi-square tests were used for betweengroup comparisons. Age was reported as means \pm standard deviations (SD), medians, and ranges in months. The difference in age between GJB2 and SLC26A4 positive subgroups was tested using the Mann-Whitney $\mathrm{U}$ test.

Confidence intervals were computed using the Clopper-Pearson method. A $P$ value of less than 0.05 was considered statistically significant. Statistical analysis was performed with IBM SPSS Statistics, version 24 (SPSS).

\section{Study population}

\section{RESULTS}

From 2012 to 2017, we successfully conducted genetic screening of 1,172,234 newborns, finding 360 (0.03\%) positive, $2638(0.23 \%)$ at-risk, 52,979 (4.52\%) inconclusive, and $1,116,257(95.22 \%)$ negative results (Supplementary Table S2 online).

To evaluate outcomes of genetic screening, we identified 55,977 newborns (4.78\%) harboring at least one of the 
Table 1 Characteristics of the 12,778 subjects who participated in follow-up interviews

Characteristic

\begin{tabular}{|c|c|}
\hline All & $12,778(100)$ \\
\hline \multicolumn{2}{|l|}{ Sex } \\
\hline Male & $6,920(54.2)$ \\
\hline Female & $5,703(44.6)$ \\
\hline Unspecified & $155(1.2)$ \\
\hline \multicolumn{2}{|l|}{ Age at follow-up (in months) } \\
\hline 2 & $453(3.5)$ \\
\hline $3-5$ & $5,031(39.4)$ \\
\hline $6-11$ & $4,611(36.1)$ \\
\hline $12-17$ & $2,303(18.0)$ \\
\hline $18-23$ & $91(0.7)$ \\
\hline$>24^{\mathrm{a}}$ & $289(2.3)$ \\
\hline \multicolumn{2}{|l|}{ Risk factors ${ }^{b}$} \\
\hline None & $11,108(86.9)$ \\
\hline $\mathrm{NICU}$ & $1,177(9.2)$ \\
\hline Family history of early-onset hearing loss ${ }^{c}$ & $491(3.8)$ \\
\hline Family history with ototoxic medications & $57(0.4)$ \\
\hline Consanguinity & $5(<0.1)$ \\
\hline Ototoxic medications during pregnancy & $2(<0.1)$ \\
\hline Neonatal bacterial meningitis ${ }^{d}$ & $1(<0.1)$ \\
\hline
\end{tabular}

NICU neonatal intensive care unit.

aThe oldest child was 56 months old.

bSubjects with two or more risk factors were calculated separately. In total, 1,670 (13.1\%) had risk factors.

Individuals reporting hearing loss with age in elderly were not considered as a family history.

dOne neonate was infected with bacterial meningitis but not admitted to NICU. screened variants (Fig. 1), including 32,474 with contact information for phone interviews. We contacted 19,125 from both interview periods combined, and 12,778 (66.81\%; mean age $=8.1 \pm 5.7$ months, median age $=6$ months, range 2-56 months) were eligible for the primary analysis, including $112(0.9 \%)$ positive, 11,591 (90.7\%) inconclusive, and 1075 $(8.4 \%)$ at-risk for ototoxicity. By the time of the last interviews, $107(0.8 \%)$ subjects were reported to have hearing loss (Fig. 1).

Table 1 shows the demographic characteristics of the 12,778 subjects. About $79.0 \%$ were younger than one year old, and $54.2 \%$ were male. Most subjects (86.9\%) reported no risk factor for hearing loss, and 1,177 (9.2\%) infants had been admitted to a neonatal intensive care unit.

\section{Benefits of genetic screening At-risk for ototoxicity}

Genetic screening identified 2,638 ( $0.23 \%$ of total) newborns predisposed to ototoxicity undetectable by hearing screening. Follow-up investigations revealed 57 subjects with a selfreported family history of drug-induced hearing loss (Table 1). Of these, 41 subjects had $M T-R N R 1$ variants, and 98\% (40/41) had a family history consistent with maternal inheritance, which was significantly enriched compared with those without $M T-R N R 1$ variants $(31 \%, 5 / 16)(p<0.001)$ (Supplementary Table S3 online).

\section{Stratification of newborns by genotypes}

Hearing screening results were compared among groups stratified by genetic screening results (Fig. 2). The refer rate of

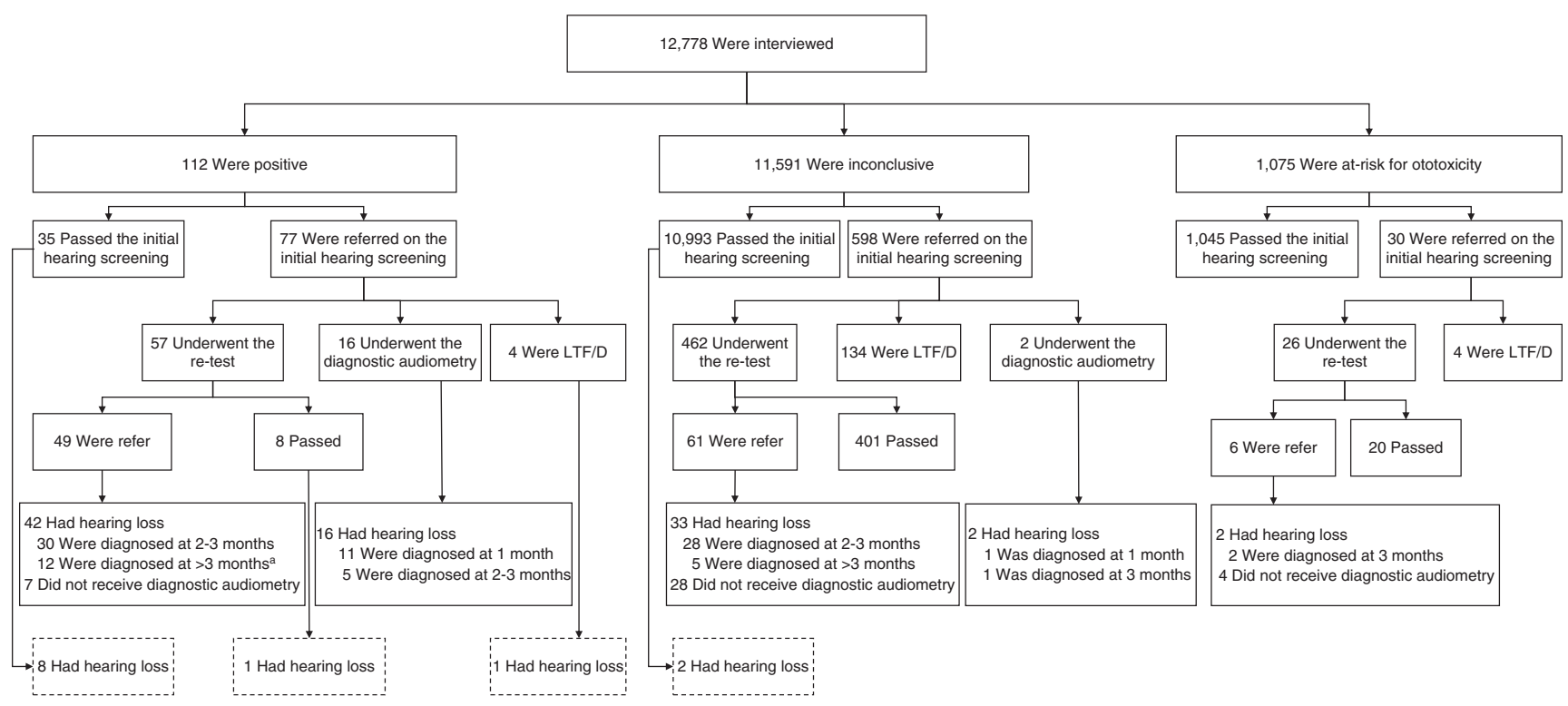

Fig. 2 Hearing screening results of 12,778 newborns with a non-negative genetic screening result who participated in follow-up interviews. Dotted box

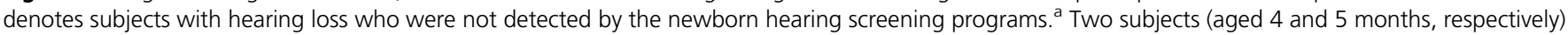
who had not had diagnostic audiometry but who were behaviorally diagnosed with severe/profound hearing loss were categorized in this group. LTF/D lostto-follow-up/documentation. 


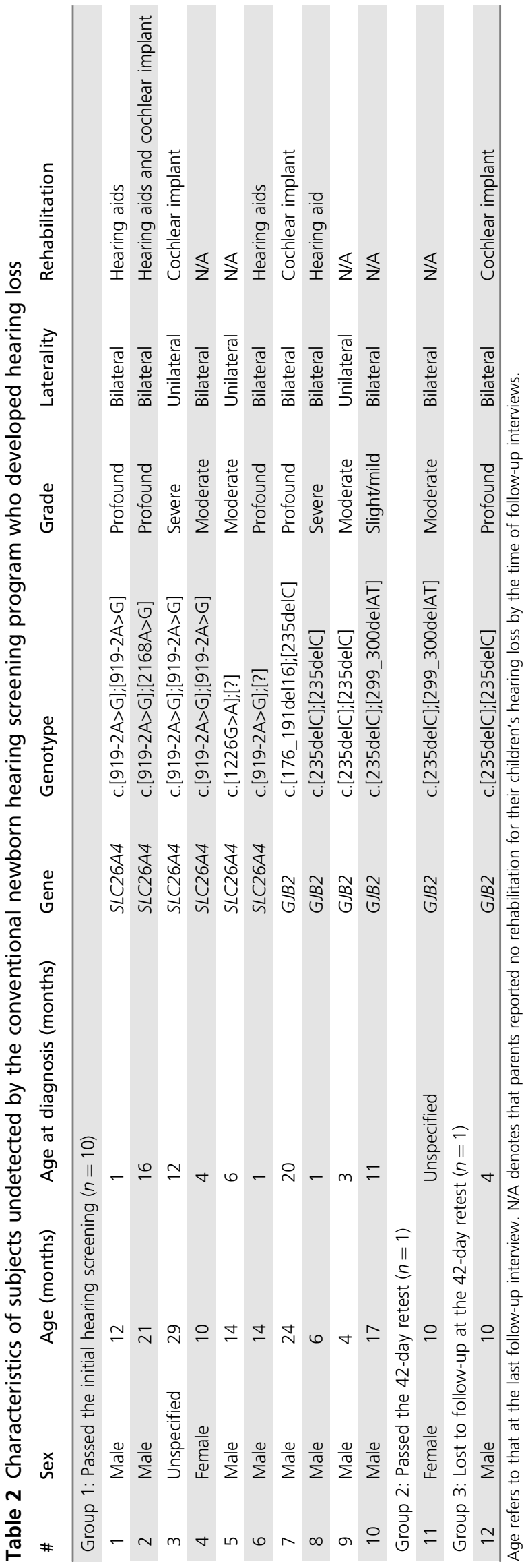

the initial hearing screening was $69 \%$ (77/112, 95\% confidence interval [CI] 59-77) in the positive group, which was significantly higher than $5.2 \%(598 / 11,591,95 \%$ CI, 4.8-5.6, $P<0.001)$ and $2.8 \%(30 / 1,075,95 \% \mathrm{CI}, 1.9-4.0, P<0.001)$ in the inconclusive and at-risk groups, respectively. The refer rate of the 42-day retest was even higher in the positive group $(86 \%, 49 / 57,95 \%$ CI, $74-94)$ than in both inconclusive $(13 \%$, $61 / 462,95 \% \mathrm{CI}, 10-17, P<0.001)$ and at-risk $(23 \%, 6 / 26,95 \%$ CI, 9-44, $P<0.001)$ groups, respectively.

Overall, among referrals from the initial hearing screening, $46 / 77$ (60\%, 95\% CI, 48-71) in the positive group were diagnosed with hearing loss by three months of age, which was significantly higher than the rates in both inconclusive $(5.0 \%, 30 / 598,95 \% \mathrm{CI}, 3.4-7.1, P<0.001)$ and at-risk (7\%, 2/30, 95\% CI, 0.8-22, $P<0.001)$ groups (Fig. 2).

Subjects with different genetic results were compared to evaluate the relationship between genotypes and rates of LTF/ D. The rate of LTF/D was significantly lower in the positive group $(5 \%, 4 / 77,95 \% \mathrm{CI}, 1-13)$ than in the inconclusive group $(22 \%, 134 / 598,95 \% \mathrm{CI}, 19-26, P<0.001)$, but there was no significant difference between the at-risk and inconclusive groups $(13 \%[4 / 30]$ vs. $22 \%, P=0.24)$.

Sixteen subjects in the positive group referred from initial hearing screening underwent diagnostic audiologic evaluation directly, bypassing the 42-day retest. Eleven were diagnosed with permanent hearing loss at one month of age, earlier than the average time to diagnosis, typically after the 3-month diagnostic audiometry. The remaining 5 and 30 other subjects in the positive group rescreened by 42 -day retest were diagnosed with permanent hearing loss by three months of age (Fig. 2).

\section{Hearing loss cases not detected by hearing screening}

Among the cohort eligible for the primary analysis, $107 \mathrm{sub}-$ jects were reported to have hearing loss (Fig. 1) by the time of follow-up interviews (mean age $=9.5 \pm 8.7$, median age $=$ 7 months, range 2-56 months). Of these, only 95 were diagnosed following the protocol of the conventional NBHS program (Fig. 2), indicating a 13\% (12/95) increase in identifying children in need of early intervention. The remaining 12 subjects (10 positive and 2 inconclusive) fell into three categories: 10 passed the initial hearing screening, 1 passed the 42-day retest, 1 was LTF/D (Table 2). None of these 12 subjects would have been identified by risk factor-indicated audiologic monitoring.

\section{Genotype-phenotype correlation}

The secondary analysis was focused on the genotype-phenotype correlation in 107 subjects confirmed to have hearing loss by the time of follow-up interviews (Table 3).

Of 112 subjects in the positive group, 68 (61\%, 95\% CI, 51-70) were reported to have hearing loss, including 46 diagnosed at 3 months or earlier by NBHS, 12 identified by NBHS but diagnosed later than 3 months, and 10 identified solely based on genetic screening results (Fig. 2). Mean ages for GJB2 and SLC26A4 positive subgroups were not significantly different (10.4 \pm 9.6 vs. $11.0 \pm 9.3$ months, $P=0.60)$. However, 


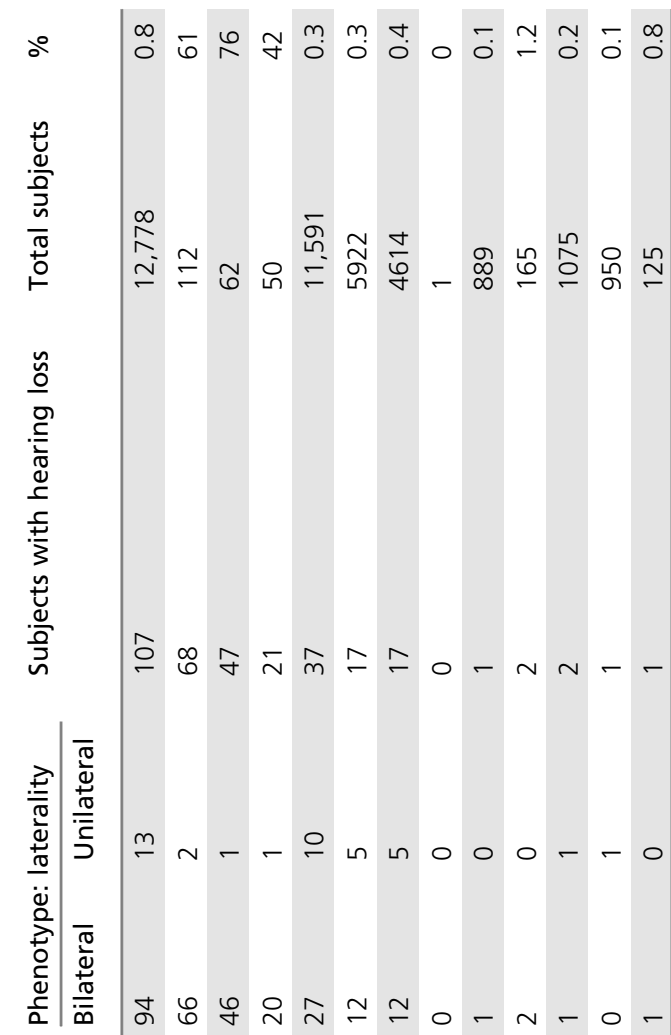

s.

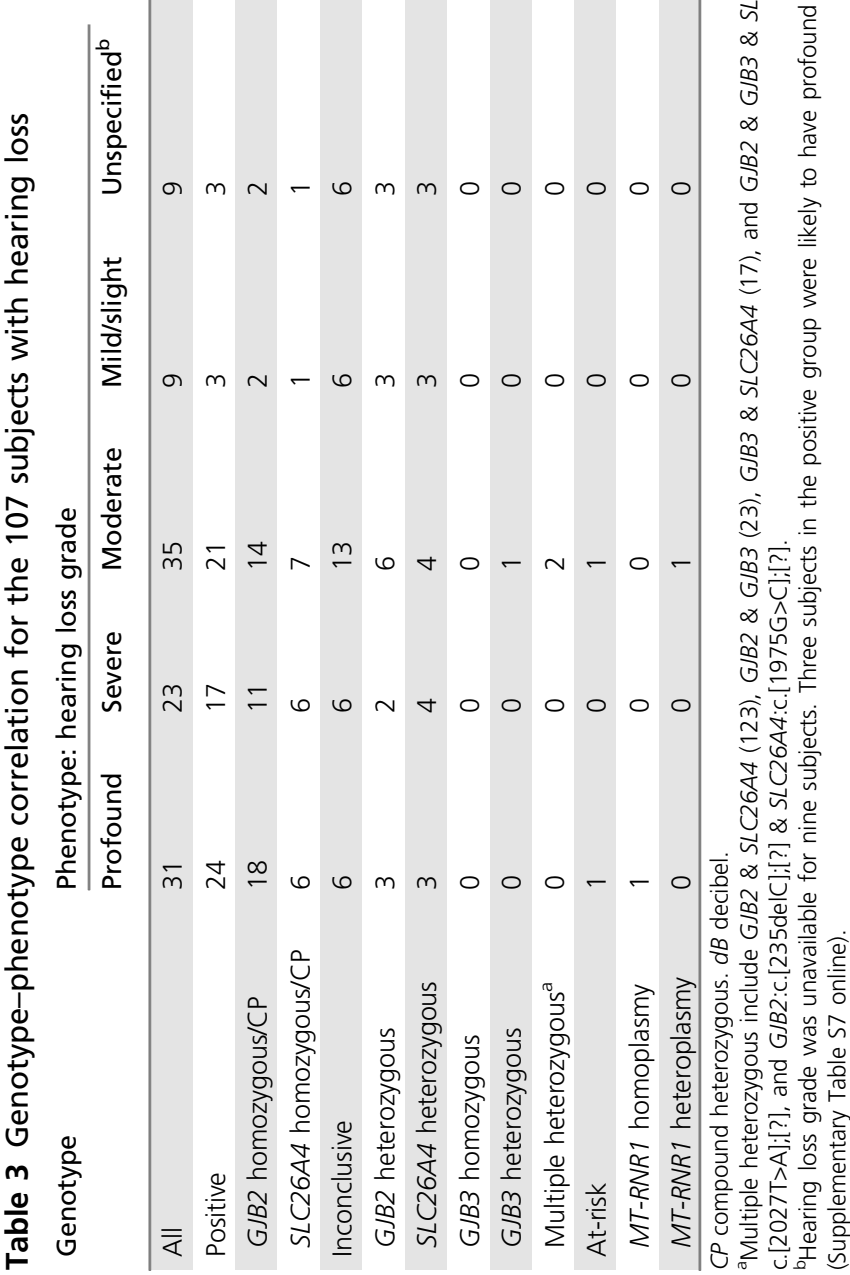

$76 \%$ (95\% CI, 63-86) of subjects with a positive GJB2 result were reported to have hearing loss, whereas the rate was $42 \%$ (95\% CI, 28-57) in the SLC26A4 positive subgroup $(P<0.001)$. Both subgroups had predominantly bilateral hearing loss (46/ $47,98 \%$ and $20 / 21,95 \%$, respectively). Moreover, subjects in the positive group were more likely to have profound or severe hearing loss $(60 \%)$ compared with those in the inconclusive group $(32 \%)(P=0.006)$ (Table 3$)$.

Of 11,591 subjects in the inconclusive group, $37(0.3,95 \%$ CI, 0.2-0.4) were reported to have hearing loss, slightly higher than but not significantly different from the population prevalence. Of one GJB3 homozygote (age 13 months) and 889 GJB3 heterozygotes (mean age $=8.1 \pm 5.5$ months, median age $=7$ months, range $2-44$ months), only one GJB3 heterozygote was diagnosed with hearing loss (Supplementary Table S4 online). None of the 25 GJB2/GJB3 double heterozygotes (mean age $=7.6 \pm 3.9$ months) reported hearing loss at the time of the last follow-up interviews (Supplementary Table S5 online). Hearing loss family history was not enriched in subjects with GJB3 variants $(3.1 \%, 28$ / $915)$ over those without $(4.1 \%, 463 / 11,372, P=0.23)$.

Of 1,075 subjects in the at-risk group, only 2 had hearing loss (Supplementary Table S6 online). Neither of them was reported to have had any exposure to ototoxic drugs; therefore, the mitochondrial variant identified was unlikely the cause of hearing loss in either case.

\section{DISCUSSION}

Herein we report results of a large-scale genetic newborn screening program for risk of hearing loss in China. The goal of genetic screening was not to diagnose all hereditary deafness but rather to supplement hearing screening to ensure that children with permanent hearing loss receive timely identification and intervention. We chose a limited screening panel for population genetic screening to achieve the goal, because it has the advantages of low-cost, fast turnaround time, easy interpretation, and low uncertainty of results over comprehensive diagnostic testing methods. Even with just the limited panel, our study has already demonstrated benefits of genetic screening, including elucidating etiologies, discerning high-risk subgroups, identifying additional children who may benefit from early intervention, and informing at-risk newborns and their maternal relatives of increased susceptibility to ototoxicity.

Our primary analysis demonstrates that genetic screening informed $0.23 \%$ of newborns and their maternal family members of increased susceptibility to potentially preventable ototoxicity due to MT-RNR1 variants by avoiding exposure to aminoglycoside antibiotics. ${ }^{13}$ Notably, all subjects from 41 families with a self-reported family history of drug-induced hearing loss had not had exposure to aminoglycoside antibiotics and none of them developed hearing loss, as family members were informed of the genetic risk of ototoxicity and received genetic counseling.

Incorporating genetic screening into the NBHS program can identify additional newborns with hearing loss at an 
earlier age. ${ }^{14-16}$ Our data quantified a $13 \%$ (12/95) improvement in detection rate of hearing loss by three months of age conferred by a limited genetic screening panel. Furthermore, although population screening and long-term follow-up are required to elucidate the exact penetrance of positive genotypes identified by our screening test, it is assumed to be nearly complete, because, to our knowledge, clinically confirmed normal hearing adults with such genotypes have not been reported in the literature. Therefore, all 112 subjects with a positive genetic result are anticipated to develop hearing loss eventually. Of these 112, 47 subjects were not detected by the conventional NBHS program (35 passed the initial inpatient NBHS, 8 passed the 42 -day retest, and 4 were LTF/D), and the remaining 65 subjects were detected by hearing screening alone, suggesting a 72\% (47/65) potential improvement of the detection yield in the positive group. Notably, only 10 of 47 (21\%) subjects would be detected by risk factor-indicated audiologic monitoring. Given the $0.03 \%$ genetically positive rate, the $58 \%$ diagnostic yield of hearing screening in the positive group, and the $0.1-0.3 \%$ prevalence of congenital hearing loss in the general population, our genetic screening is estimated to detect $6-17 \%$ of all newborns with hearing loss detectable by hearing screening and another $4-13 \%$ that would have been undetected. Hence, the benefit of genetic screening is remarkable, compared with a $<1 \%$ additional yield by risk factor-indicated audiologic monitoring recommended by the Joint Committee on Infant Hearing. $^{17-19}$

LTF/D undermines the clinical effectiveness of NBHS programs. ${ }^{20}$ We report that the LTF/D rate was significantly lower in the positive group (5\%) than in the inconclusive group $(22 \%)$. The lower LTF/D in the positive group may be attributed to the intrinsic high risk of hereditary hearing loss and to genetic counseling, which highlighted the importance of follow-up. Notably, one of four subjects in the positive group who were LTF/D by the conventional NBHS program developed hearing loss by the time of the last interview, and the remaining were predicted to be likely to develop hearing loss later in life and were aware of the risk from the benefit of genetic screening. Hence, incorporating genetic screening into the NBHS program effectively identifies the high-risk population who need active surveillance and who can benefit the most from early intervention for hearing loss.

Genetic screening can decrease the time to diagnosis and intervention in the high-risk population. The time to diagnosis depends on availability of genetic screening results as well as the clinical practice upon receiving such results, which varied in different hospitals and could be further optimized. Some subjects in the positive group were diagnosed by one month of age, much earlier than the typical age of diagnosis after 3-month diagnostic audiometry, 4,21 because genetic results informed them to bypass the 42-day retest and proceed directly to diagnostic audiometry. Furthermore, a positive genetic result helps establish an etiologic diagnosis of permanent hearing loss, eliminating the need for repeated audiologic evaluations in cases with borderline results that delay diagnosis. Among refers from the initial hearing screening, $60 \%$ of newborns in the positive group were diagnosed with hearing loss by three months of age, suggesting that subjects should schedule an audiologic evaluation directly upon receipt of a positive genetic result. As a comparison, although very few subjects in the inconclusive group underwent the diagnostic audiologic evaluation directly and received an earlier diagnosis as well, only 6\% (33/598) of subjects who were referred from the initial hearing screening developed hearing loss by the time of the last interviews. Therefore, to avoid unnecessary anxiety and medical costs, it is not recommended that newborns with an inconclusive genetic screening result bypass the 42-day retest. While offering audiologic evaluation before one month of age to all initial refers would in theory decrease the time to diagnosis, it would not be an effective use of public health resources due to a high false positive rate of the initial hearing screening. Limited genetic screening is able to discern the high-risk positive group and provide an etiologic diagnosis, so that resources can be effectively prioritized to the early diagnosis and intervention of those who truly need them.

Our secondary analysis focused on genotype-phenotype correlations. By the time of follow-up interviews, 44 subjects in the positive group aged 3 to 38 months were reported to be hearing based on their latest audiologic evaluation or behavioral observation. They might have delayed-onset or subclinical hearing loss. Long-term longitudinal follow-up of the hearing status of individuals with high-risk genotypes is necessary to elucidate the genotype-specific natural history and penetrance of hearing loss. ${ }^{8}$

In the inconclusive group, 37 subjects were reported to have hearing loss. Of note, GJB2 or SLC26A4 heterozygotes might have a second pathogenic allele in the same gene not screened by our panel. Identifying the second allele in these subjects would not only provide an etiologic diagnosis and be of value in genetic counseling, ${ }^{22}$ but also inform future panel design of recurrent alleles with relatively high frequencies. Nevertheless, our data did not provide evidence to support the pathogenicity of GJB3 variants in autosomal recessive, dominant, or digenic hearing loss, consistent with a recent study. ${ }^{23}$ Although their roles in late-onset recessive or digenic hearing loss cannot be determined, the lack of enrichment of hearing loss family history in subjects with these variants indicates they do not cause late-onset dominant hearing loss.

The benefits of genetic screening could be enhanced further by optimizing ethnicity-specific panels and increasing the number of variants without increasing the cost and turnaround time. The current screening panel was designed in 2011 when large population databases with ethnicity-specific allele frequencies were unavailable. We now observe GJB2: c.35delG, GJB2:c.167delT, and SLC26A4:c.2162C>T with high frequencies in European, Jewish, and Latino populations, respectively, in the Genome Aggregation Database, ${ }^{24}$ but very low or even undetected in our Chinese study population (Supplementary Table S1 online). Replacing these three variants and the GJB3 variants with other relatively common 
pathogenic variants in the Chinese population is anticipated to improve the panel.

Strengths of this study are a large sample size and outcome assessment with sufficient power to demonstrate the benefits of genetic screening. It provides evidence and experience for other countries, hospitals, and laboratories to implement such strategies to meet the urgent need of improving NBHS.

The weaknesses include vague recalls from some interviewees and insufficient long-term follow-up to assess intervention outcomes, which warrant further investigations. Furthermore, cost-effectiveness analysis of incorporating genetic screening into newborn hearing screening program was not conducted in this study. Finally, we were not able to follow up newborns with negative genetic screening results as a better comparator.

In conclusion, this study provides convincing evidence that incorporating a limited genetic screening panel is an effective strategy to improve the effectiveness of newborn hearing screening programs by elucidating etiologies, discerning highrisk subgroups for vigilant management, identifying additional children who may benefit from early intervention, and informing at-risk newborns and their maternal relatives of increased susceptibility to ototoxicity. Our results call for rigorous clinical trials to evaluate the cost-effectiveness and long-term benefits of incorporating genetic screening into newborn hearing screening programs.

\section{SUPPLEMENTARY INFORMATION}

The online version of this article (https://doi.org/10.1038/s41436019-0481-6) contains supplementary material, which is available to authorized users.

\section{ACKNOWLEDGEMENTS}

This study was supported by Shenzhen Engineering Laboratory for Innovative Molecular Diagnostics (DRC-SZ[2016]884 to S.Z.); by grants from the National Natural Science Foundation of China (Major Project numbers 81530032 and 81830028 to Q.W.); by a grant from the National Institutes of Health/the National Institute on Deafness and Other Communication Disorders (R01DC015052 to C.C.M. and J. Shen); by a grant from the National Natural Science Foundation of China (number 81600690 to C.S.); and by the National Institute for Health Research (NIHR) Manchester Biomedical Research Centre (to C.C.M.).

\section{DISCLOSURE}

J.X., C.Y., Y. Yin, Lijian Zhao, S.Z., J. Sun, Y. Yang, W.W., X.W., J. Zhang, J. Zou, H. Luo, Y.H., W.Y., H.Z., J.W., H.Y., X.X., and Z.P. were employed at $\mathrm{BGl}$ at the time of submission. C.C.M. and J. Shen worked for pay for service diagnostic laboratories providing genetic testing. The other authors declare no conflicts of interest.

Publisher's note: Springer Nature remains neutral with regard to jurisdictional claims in published maps and institutional affiliations.

\section{REFERENCES}

1. Kennedy CR, McCann DC, Campbell MJ, et al. Language ability after early detection of permanent childhood hearing impairment. $\mathrm{N}$ Engl J Med. 2006;354:2131-2141.

2. Patel H, Feldman M. Universal newborn hearing screening. Paediatr Child Health. 2011;16:301-305.

3. Linden Phillips L, Bitner-Glindzicz M, Lench $N$, et al. The future role of genetic screening to detect newborns at risk of childhood-onset hearing loss. Int J Audiol. 2013;52:124-133.

4. Nelson HD, Bougatsos C, Nygren P, 2001 US Preventive Services Task Force. Universal newborn hearing screening: systematic review to update the 2001 US Preventive Services Task Force Recommendation. Pediatrics. 2008;122:e266-276.

5. Morton CC, Nance WE. Newborn hearing screening-a silent revolution. N Engl J Med. 2006;354:2151-2164.

6. Subbiah K, Mason CA, Gaffney M, Grosse SD. Progress in documented early identification and intervention for deaf and hard of hearing infants: CDC's hearing screening and follow-up survey, United States, 2006-16. J Early Hear Detect Interv. 2018;3:1-7.

7. Zhang J, Wang P, Han B, et al. Newborn hearing concurrent genetic screening for hearing impairment-a clinical practice in 58,397 neonates in Tianjin, China. Int J Pediatr Otorhinolaryngol. 2013;77:1929-1935.

8. Wu CC, Tsai $\mathrm{CH}$, Hung CC, et al. Newborn genetic screening for hearing impairment: a population-based longitudinal study. Genet Med. 2017;19:6-12.

9. Peng Q, Huang S, Liang $Y$, et al. Concurrent genetic and standard screening for hearing impairment in 9317 Southern Chinese newborns. Genet Test Mol Biomarkers. 2016;20:603-608.

10. Wang QJ, Zhao $Y L$, Rao SQ, et al. Newborn hearing concurrent gene screening can improve care for hearing loss: a study on 14,913 Chinese newborns. Int J Pediatr Otorhinolaryngol. 2011;75:535-542.

11. Li SX, Chen DL, Zhao SB, et al. Cordblood-based high-throughput screening for deafness gene of 646 newborns in Jinan area of China. Clin Exp Otorhinolaryngol. 2015;8:211-217.

12. World Health Organization. Grades of hearing impairment. http://www. who.int/pbd/deafness/hearing_impairment_grades/en/. Accessed 13 February 2018.

13. Barbarino JM, McGregor TL, Altman RB, Klein TE. PharmGKB summary: very important pharmacogene information for MT-RNR1. Pharmacogenet Genomics. 2016;26:558-567.

14. Minami SB, Mutai $H$, Nakano A, et al. GJB2-associated hearing loss undetected by hearing screening of newborns. Gene. 2013;532:41-45.

15. Norris VW, Arnos KS, Hanks WD, et al. Does universal newborn hearing screening identify all children with GJB2 (Connexin 26) deafness? Penetrance of GJB2 deafness. Ear Hear. 2006;27:732-741.

16. Akinpelu OV, Peleva E, Funnell WR, Daniel SJ. Otoacoustic emissions in newborn hearing screening: a systematic review of the effects of different protocols on test outcomes. Int J Pediatr Otorhinolaryngol. 2014;78:711-717.

17. American Academy of Pediatrics, Joint Committee on Infant Hearing. Year 2007 position statement: principles and guidelines for early hearing detection and intervention programs. Pediatrics. 2007;120:898-921.

18. Beswick R, Driscoll C, Kei J, Glennon S. Targeted surveillance for postnatal hearing loss: a program evaluation. Int J Pediatr Otorhinolaryngol. 2012;76:1046-1056.

19. Appelbaum EN, Howell JB, Chapman D, et al. Analysis of risk factors associated with unilateral hearing loss in children who initially passed newborn hearing screening. Int J Pediatr Otorhinolaryngol. 2018; 106:100-104.

20. Alam S, Gaffney M, Eichwald J. Improved newborn hearing screening follow-up results in more infants identified. J Public Health Manag Pract. 2014;20:220-223.

21. Thompson DC, McPhillips $H$, Davis RL, et al. Universal newborn hearing screening: summary of evidence. JAMA. 2001;286:2000-2010.

22. Alford RL, Arnos KS, Fox M, et al. American College of Medical Genetics and Genomics guideline for the clinical evaluation and etiologic diagnosis of hearing loss. Genet Med. 2014;16:347-355.

23. Huang S, Huang B, Wang G, et al. The relationship between the GJB3 C.538C > T variant and hearing phenotype in the Chinese population. Int J Pediatr Otorhinolaryngol. 2017;102:67-70.

24. Lek M, Karczewski KJ, Minikel EV, et al. Analysis of protein-coding genetic variation in 60,706 humans. Nature. 2016;536:285-291. 